\title{
Excess mortality from COVID-19: a commentary on the Italian experience
}

\author{
Paolo Pasquariello ${ }^{1}$ Saverio Stranges ${ }^{2,3,4}$ []
}

Received: 7 May 2020 / Revised: 16 May 2020 / Accepted: 23 May 2020/Published online: 28 May 2020

(C) Swiss School of Public Health (SSPH+) 2020

Keywords COVID-19 - Case fatality rate - Italy · Testing · Health care system · Demographics · Comorbidites · Epidemiological trends

There has been much discussion about why case fatality rate from COVID-19 in Italy (at $13.3 \%$ as of April 20, 2020 , versus a global case fatality rate of $6.9 \%$; Worldometer 2020) is considerably larger than in other countries. In this commentary, we propose several potential explanations underlying these differences.

\section{Demographics and chronic comorbidities}

As widely reported, the novel Severe Acute Respiratory Syndrome CoronaVirus-2 (SARS-CoV-2) appears to be most harmful to male elderly (Onder et al. 2020) and Italy has one of the oldest populations globally. In addition, Italy's elderly are well-integrated within society. For instance, Italian elderly usually live with their extended, multi-generation families, and actively participate in the life of their communities (Mazzola et al. 2016). While socially beneficial, this integration may have facilitated the exposure of this vulnerable population subgroup to infection more than in other high-income countries. Accordingly, the distribution of the demographic profile of COVID-19 deceased individuals in Italy is skewed, with

Saverio Stranges

saverio.stranges@uwo.ca

1 Ross School of Business, University of Michigan, Ann Arbor, MI 48109, USA

2 Department of Epidemiology and Biostatistics, Schulich School of Medicine and Dentistry, Western University, Kresge Building, Room K201B, London, ON N6A 5C1, Canada

3 Department of Population Health, Luxembourg Institute of Health, Strassen, Luxembourg

4 Humanitas University Medical School, Milan, Italy median age of around 81 years (Istituto Superiore di Sanita' 2020).

COVID-19 appears to be especially harmful to individuals with preexisting chronic conditions or high-risk behaviors (smoking), some of which are highly prevalent in the Italian population, especially among the elderly (Onder et al. 2020). Accordingly, hypertension, type 2 diabetes, ischemic heart disease, atrial fibrillation, cancer, chronic kidney disease, stroke and COPD are among the most common comorbidities consistently associated with COVID-19-related Italian fatalities (Onder et al. 2020; Istituto Superiore di Sanita' 2020).

\section{Definition of COVID-19 related deaths}

In the absence of consistent international guidelines, there may be considerable discrepancies among countries in distinguishing deaths from other causes while being infected with COVID-19. To our understanding, Italy currently labels COVID-19-related deaths as those where patients tested positive to the virus, independent of the primary underlying cause of morbidity. Other countries may be using a less expansive definition of COVID-19related deaths, as those induced directly by the virus, thus reporting lower case fatality rates.

\section{Effect of COVID-19 on the Italian National Health System}

The coronavirus pandemic severely stressed the local provision of health care. In particular, intensive care units (ICUs) of local and regional hospitals in Italy in general, and especially in the Lombardy region, had to deal with 
thousands of patients with severe acute respiratory syndromes induced by COVID-19. This nearly exhausted the available provision not only of ICU beds, but also of respirators and ventilators (Rosenbaum 2020). In addition, the epidemic progressed at a speed that made adding capacity challenging while the global supply chain is stretched and at times broken. This suggests that some patients may have not received the best possible care, leading to worse health outcomes. These events are remarkable, since Lombardy is one of the wealthiest regions in Europe, while the Italian Health System is widely considered among the best in the world in terms of access, quantity and quality of care provided (World Population Review 2020).

It is equally important to emphasize that COVID-related case fatality rates within Italy display significant heterogeneity, e.g., as high as $18.5 \%$ in Lombardy, also the most populous region of Italy, and as low as $4.3 \%$ in Umbria, among the least populated, as of April 20, 2020 (Dipartimento della Protezione Civile 2020). Yet, the regional case fatality rate is also highly positively correlated (up to 68\%) with the regional-level number of hospitalized symptomatic and/or ICU-admitted COVID-19 patients, once again suggesting that fatalities spiked as hospitals' capacity diminished, especially in the most affected northern regions where the vast majority of deaths occurred.

\section{Extent of testing for COVID-19 and the tip of the iceberg}

Italy and South Korea are among the countries where the most tests per thousand people for COVID-19 were performed as of April 20, 2020 (Our World In Data 2020). Of course, running more tests implies in principle that a larger portion of the coronavirus iceberg would be observed, including infected but asymptomatic individuals, relative to other countries where COVID-19 is stealthily spreading and killing. However, Italy has so far mostly tested symptomatic people, while it was widely reported that South Korea adopted extensive contact tracing by testing several asymptomatic people for each symptomatic one on the basis of immediate proximity. Nevertheless, this approach (adopted by other Asian countries as well) also raises concerns for the potential violation of privacy and human rights; hence, its applicability to Western countries is uncertain.

In light of these observations, we developed a simple model to estimate the Italian coronavirus under-testing and iceberg as a function of recent estimates of the asymptomatic proportion of infected individuals (Nishiura et al. 2020). Intuitively, our model is based on expressing basic epidemiological aggregates as explicit functions of observable and unobservable parameters from the current
Italian and South Korean testing experiences as well as on assuming that Italy would have experienced similar observable epidemiological outcomes as South Korea if it had adopted the same testing approach. See Pasquariello and Stranges (2020) for further details and analysis.

We estimate from this model that if Italy had followed the more proactive South Korean testing approach, it should have tested between ten and more than twenty asymptomatic people for each symptomatic one it did test. Such more widespread testing would have yielded a coronavirus iceberg from one-tenth to more than two times larger than its observed tip. These estimates translate in non-trivial over-reporting of the current COVID-19 case fatality rate in Italy, as compared to countries (like South Korea) adopting proactive and extensive contact tracing. In particular, our model implies an estimated case fatality rate of slightly less than $9 \%$, i.e., about one-third lower than the current one.

\section{Lessons learned from the Italian experience}

We conclude that Italy's COVID-19 case fatality rate is likely to be inflated by such factors as the iceberg effect of under-testing. However, we also acknowledge that deaths in Italy are likely higher than in other equally affected countries because of its unique demographic and socioeconomic profile. In addition, we emphasize the central role played by the stress imposed by the pandemic on the health care system of the most affected areas and its likely contribution to their high death tolls.

Therefore, we caution from ignoring the lessons that the Italian circumstances may offer to other impacted Western countries. The COVID-19 pandemic has exposed several intrinsic structural limitations of the Italian national public health care system, especially a lack of coordinated and timely response mechanisms for enabling rapid actions, like those deployed by several Asian countries, and a lack of adequate community response, which is critical for epidemic containment.

In a context of limited evidence and significant uncertainty, Italy is running a massive public health intervention that is unique in the history of Western democracies. We all have to hope that it succeeds.

Funding This article was not funded by any grant.

\section{Compliance with Ethical Standards}

Conflict of interest The authors declare that they have no conflict of interest. 
Human and animal rights This article does not contain any studies with human participants or animals.

\section{References}

Dipartimento della Protezione Civile (2020) Emergenza coronavirus, dati regionali. https://github.com/pcm-dpc/COVID-19/blob/mas ter/dati-regioni/dpc-covid19-ita-regioni.csv. Accessed $21 \mathrm{Apr}$ 2020

Istituto Superiore di Sanita' (2020) Characteristics of COVID-19 patients dying in Italy-report based on available data on April 23, 2020. https://www.epicentro.iss.it/en/coronavirus/bollettino/ Report-COVID-2019_23_april_2020.pdf

Mazzola P, Rimoldi S, Rossi P et al (2016) Aging in Italy: the need for new welfare strategies in an old country. The Gerontologist. https://doi.org/10.1093/geront/gnv152

Nishiura H, Kobayashi T, Miyama T et al (2020) Estimation of the asymptomatic ratio of novel coronavirus infections (COVID-19). Int J Infect Dis. https://doi.org/10.1016/j.ijid.2020.03.020
Onder G, Rezza G, Brusaferro S (2020) Case-fatality rate and characteristics of patients dying in relation to COVID-19 in Italy. JAMA. https://doi.org/10.1001/jama.2020.4683

Our World In Data (2020) COVID-19 testing. https://ourworldindata. org/covid-testing. Accessed 21 Apr 2020

Pasquariello P, Stranges S (2020) Excess mortality from COVID-19: lessons learned from the Italian experience. Preprints. https://doi. org/10.20944/preprints202004.0065.v2

Rosenbaum L (2020) Facing Covid-19 in Italy_ethics, logistics, and therapeutics on the epidemic's front line. NEJM. https://doi.org/ 10.1056/NEJMp2005492

World Population Review (2020) Best healthcare in the world 2020. https://worldpopulationreview.com/countries/best-healthcare-inthe-world/. Accessed 21 Apr 2020

Worldometer (2020) COVID-19 coronavirus pandemic. https://www. worldometers.info/coronavirus/. Accessed 21 Apr 2020

Publisher's Note Springer Nature remains neutral with regard to jurisdictional claims in published maps and institutional affiliations. 